\title{
Rat vibrissa dermal papilla cells promote healing of spinal cord injury following transplantation
}

\author{
MEIYING LI ${ }^{1,2^{*}}$, XIANGLIN MEI ${ }^{3,4^{*}}$, SHUANG LV $^{1}$, ZECHUAN ZHANG $^{5}$, JINYING XU ${ }^{1}$, \\ DONGJIE SUN ${ }^{1}$, JIAYI XU ${ }^{1}$, XIA HE ${ }^{1}$, GUANGFAN CHI ${ }^{1}$ and YULIN LI ${ }^{1}$ \\ ${ }^{1}$ Key Laboratory of Pathobiology, Ministry of Education, Jilin University; \\ ${ }^{2}$ Department of Surgery, The First Hospital of Jilin University, Changchun, Jilin 130021; \\ ${ }^{3}$ Department of Pathology, The Second Hospital of Jilin University, Changchun, Jilin 130041; \\ ${ }^{4}$ National Engineering Laboratory for Druggable Gene and Protein Screening, Northeast Normal University, \\ Changchun, Jilin 130024; ${ }^{5}$ Clinical Medical College, Jilin University, Changchun, Jilin 130021, P.R. China
}

Received February 18, 2017; Accepted January 18, 2018

DOI: $10.3892 /$ etm.2018.5916

\begin{abstract}
Bone marrow mesenchymal stem cell (BMSC) transplantation is effective for repairing spinal cord injuries (SCIs); however, there are limitations of clinical BMSC applications. Previously, we reported that dermal papilla cells (DPCs) secrete brain-derived neurotrophic factor and glial cell line-derived neurotrophic factor more actively than BMSCs. To analyze the therapeutic function of DPCs in SCI, primary DPCs and BMSCs were cultured from the same green fluorescence protein-transgenic rat. The cells were suspended in rat-tail collagen I and transplanted separately into completely transected spinal cord lesion sites. Grafted-cell survival was examined with a small animal in vivo imaging detection system, and lesion sites were examined histochemically. In vivo
\end{abstract}

Correspondence to: Professor Guangfan Chi or Professor Yulin Li, Key Laboratory of Pathobiology, Ministry of Education, Jilin University, 126 Xinmin Street, Changchun, Jilin 130021, P.R. China

E-mail: guangfan130@jlu.edu.cn

E-mail: ylli@jlu.edu.cn

"Contributed equally

Abbreviations: BDNF, brain-derived neurotrophic factor; bFGF, basic fibroblast growth factor; BMSCs, bone marrow mesenchymal stem cells; CNTF, ciliary neurotrophic factor; DPCs, dermal papilla cells; FBS, fetal bovine serum; FGF2, fibroblast growth factor 2; FLK-1, vascular endothelial growth factor receptor 2; GDNF, glial cell line-derived neurotrophic factor; GFP, green fluorescent protein; HGF, hepatocyte growth factor; IL, interleukin; NCSCs, neural crest stem cells; NGF, nerve growth factor; NSCs, neural stem cells; NTFs, neurotrophic factors; SCI, spinal cord injury; TGF- $\beta 1$, transforming growth factor- $\beta 1$; TNF- $\alpha$, tumor necrosis factor- $\alpha$; VEGFA, vascular endothelial growth factor A

Key words: dermal papilla cells, mesenchymal stem cells, spinal cord injury, cell transplantation, regeneration imaging revealed enhanced lesion filling and survival with DPC grafts compared with BMSC grafts on days 14 and 21 post-transplantation. Hematoxylin and eosin staining demonstrated that lesion area sizes in the two groups were not markedly different. In the DPC transplant group, more axons formed within the lesion sites. CD31-positive vessel-like structures were more abundant in lesion sites near the grafted cells in the DPC group. The results of the present study suggest that DPCs may be a valuable alternative source of stem cells for autologous cell therapy for the treatment of SCI.

\section{Introduction}

Spinal cord injury (SCI) is a devastating condition, which involves the sudden loss of sensory, motor and autonomic functions distal to the areas of trauma. No effective therapy exists for treating the neurological deficits of major SCI. Stem cell-based therapies have introduced novel possibilities for the repair and restoration of neuronal functions following SCI (1-5). Transplantation of bone marrow mesenchymal stem cells (BMSCs), which represent an accessible autologous stem cell source, has reached the stage of clinical investigation and may be a promising treatment for SCI (6). According to previous reports, the mechanism underlying BMSC-induced recovery is primarily associated with the secretion of numerous cytokines and growth factors, including neurotrophic factors (NTFs), brain-derived neurotrophic factor (BDNF), glial cell line-derived neurotrophic factor (GDNF) and nerve growth factor (NGF); the angiogenic factor, vascular endothelial growth factor (VEGF) and the inflammation-associated cytokines, transforming growth factor (TGF)- $\beta 1$ and interleukin (IL)-10 (7-9). These factors likely promote endogenous repair mechanisms by stimulating neurite outgrowth and angiogenesis, and by promoting immunomodulatory effects (7-9). However, BMSC isolation is invasive, the abundance of stem cells in the bone marrow is very low, and the proliferative capacity and differentiation abilities of stem cells decrease with age (10). Due to these shortcomings, clinical applications with BMSCs are limited. Thus, there is a requirement to identify and evaluate other autologous sources of adult stem cells. 
In a previous study, we demonstrated that dermal papilla cells (DPCs) expressed mesenchymal stem cell markers, including cluster of differentiation (CD)44, CD90 and CD105, and exhibited multipotent stem cell characteristics, similar to BMSCs (11). Notably, DPCs secreted higher amounts of BDNF and GDNF, and promoted rat pheochromocytoma cell (PC12 cell) neural differentiation and axonal outgrowth more effectively compared with BMSCs in vitro. On this basis, DPCs may exhibit similar characteristics to BMSCs, including the secretion of NTFs, angiogenic factors and inflammation-associated cytokines. Thus, DPCs may potentially be utilized in autologous clinical stem cell applications for treating SCI.

In the present study, the expression of associated cytokines in DPCs and BMSCs was examined, which were isolated from the same donor rat. Thereafter, these cells were transplanted into a completely transected lesion site of the spinal cord to further investigate their therapeutic efficacies in repairing SCI in vivo.

\section{Materials and methods}

Animals. A total of 3 adult male green fluorescence protein (GFP)-transgenic SD rats (6 weeks old; 200 g) were purchased from Xing Xingming Biomedical Technology (Shanghai) Co., Ltd. (Shanghai, China) and 27 adult female SD rats (7 weeks old; $250 \mathrm{~g}$ ) were purchased from Beijing Hua Fu Kang Biotechnology Co., Ltd. (Beijing, China). All the rats were kept under identical housing conditions (temperature, $18-26^{\circ} \mathrm{C}$; humidity, $40-70 \%$ ) with a $12 \mathrm{~h}$ light-dark cycle, and given access to water and food ad libitum. All experimental procedures were approved by the Ethics Committee of Jilin University (Changchun, China) and conformed to their regulatory standards.

Isolation and cultivation of DPCs and BMSCs from GFP-transgenic rats. DPCs were isolated from the vibrissa of GFP-transgenic rats according to previously described protocols (11). Subsequently, the papillae were cultured in proliferation medium consisting of Dulbecco's modified Eagle's medium (DMEM)/Nutrient Mixture F-12 (1:1) medium (Gibco; Thermo Fisher Scientific, Inc., Waltham, MA, USA) supplemented with $10 \%$ (v/v) fetal bovine serum (FBS; HyClone; GE Healthcare Lie Sciences, Logan, UT, USA) and $10 \mathrm{ng} / \mathrm{ml}$ basic fibroblast growth factor (bFGF; PeproTech EC, Ltd., London, UK). Half of the medium was changed every 3 days. The DPCs that migrated out of the explants were digested with $0.25 \%$ trypsin (Invitrogen; Thermo Fisher Scientific, Inc.) in $0.02 \%$ ethylenediaminetetraacetic acid (Sigma-Aldrich; Merck KGaA, Darmstadt, Germany), after which they were expanded in new culture dishes at a seeding density of $1 \times 10^{4}$ cells $/ \mathrm{cm}^{2}$. The cells were passaged when $\sim 80 \%$ confluence was attained. Finally, the DPCs at passage 6 were prepared for transplantation. As a control, BMSCs were isolated from the same donor rat and cultured using a previously described method (11).

Semi-quantitative reverse transcription polymerase chain reaction $(R T-P C R)$. Total RNA from DPCs and BMSCs was extracted using TRIzol reagent (Invitrogen; Thermo Fisher Scientific, Inc.), according to the manufacturer's protocols. cDNA was synthesized from $500 \mathrm{ng}$ total RNA using the Takara RNA PCR kit (AMV) version 3.0 (Takara Biotechnology Co., Ltd. Dalian, China), only the reagents for the reverse transcription protocol were used. The reaction condition was as follows: $45^{\circ} \mathrm{C}$ for $30 \mathrm{~min}, 99^{\circ} \mathrm{C}$ for $5 \mathrm{~min}$ and $5^{\circ} \mathrm{C}$ for $5 \mathrm{~min}$. PCR was performed with $1 \mu \mathrm{l} \mathrm{cDNA}$ in a $20 \mu \mathrm{l}$ reaction volume using 2X Taq MasterMix (Beijing ComWin Biotech Co., Ltd., Beijing, China), according to the manufacturer's protocols. The reaction condition was as follows: Initial denaturation at $94^{\circ} \mathrm{C}$ for $2 \mathrm{~min}$, then 30 cycles of degeneration at $94^{\circ} \mathrm{C}$ for for $30 \mathrm{sec}$, annealing at $50-58^{\circ} \mathrm{C}$ for $30 \mathrm{sec}$ and extension at $72^{\circ} \mathrm{C}$ for $50 \mathrm{sec}$, and final extension at $72^{\circ} \mathrm{C}$ for $10 \mathrm{~min}$. PCR products were separeated by $1.5 \%$ agarose gel electrophoresis with ethidium bromide and visualized using an ultraviolet transilluminator. Sequence information for the primers used is presented in Table I.

$R T$-quantitative PCR (RT-qPCR). Total RNA from DPCs and BMSCs was extracted using TRIzol reagent, according to the manufacturer's protocols, then reverse transcribed into cDNA using the Takara RNA PCR kit (AMV) Version 3.0 (Takara Biotechnology Co.,Ltd.) according to the manufacturer's protocols. Only the reagents for the reverse transcription protocol were used. To determine the expression levels of NTFs (BDNF, GDNF, CNTF, HGF and NGF), angiogenic factor VEGF(A) and inflammation-associated cytokines [TGF- $\beta 1$, tumor necrosis factor (TNF)- $\alpha$, IL-6 and IL-1 $\beta$ ] in DPCs. qPCR was performed using TransStart ${ }^{\circledR}$ Top Green qPCR SuperMix (Beijing TransGen Biotech, Co., Ltd., Beijing, China). GAPDH was used as an internal reference to normalize mRNA expression levels. qPCR was performed in a PCR System 7300 (Applied Biosystems; Thermo Fisher Scientific, Inc.). The thermocyling conditions were as follows: $50^{\circ} \mathrm{C}$ for $2 \mathrm{~min}, 95^{\circ} \mathrm{C}$ for $10 \mathrm{~min}, 40$ cycles of $95^{\circ} \mathrm{C}$ for $15 \mathrm{sec}$ and $60^{\circ} \mathrm{C}$ for $1 \mathrm{~min}, 95^{\circ} \mathrm{C}$ for $15 \mathrm{sec}, 60^{\circ} \mathrm{C}$ for $1 \mathrm{~min}$, and $95^{\circ} \mathrm{C}$ for $15 \mathrm{sec}$. The expression levels of mRNAs were measured using the cycle-threshold values and then the results were converted to fold-changes (12). Sequence information for the primers used is presented in Table II.

Spinal cord transection and acute injection of cells. The female SD rats were randomly assigned to the DPC-transplant group $(n=10)$, the BMSC-transplant group $(n=9)$ and a control group $(n=8)$. Subsequently, the rats were deeply anesthetized by intraperitoneal injection with $10 \%$ chloral hydrate $(400 \mathrm{mg} / \mathrm{ml}$; Sigma-Aldrich; Merck KGaA). The SCI models were generated according to Medalha's method (13). Briefly, the hair over the thoracic area was shaved, the skin was disinfected with iodine and T8 vertebra were completely transected. A $\sim 1.5$-mm-long segment of spinal cord was cut using iridectomy scissors and the debris were removed by micro-aspiration. Once hemostasis was achieved, the gap was filled with $7 \mu 1$ DPCs or BMSCs $\left(10^{6}\right.$ cells total $)$ in rat-tail collagen I $(2 \mathrm{mg} / \mathrm{ml}$; Tianjin Weikai Bioeng, Ltd., Tianjin, China) using a $10-\mu$ l pipettor. As a control, $7 \mu \mathrm{l}$ collagen I without cells was microinjected into the lesion cavity. Following exposure at room temperature for $45 \mathrm{~min}$, the lesion site was covered with the fat pad, and the skin was sewn back together. Bladders were manually flushed twice daily until the animals were euthanized (on days 14 and 21 post-transplantation) and penicillin $\left(4 \times 10^{4}\right.$ units/rat; North 
Table I. Primers and annealing temperatures used for semi-quantitative polymerase chain reaction.

\begin{tabular}{|c|c|c|}
\hline Gene & Primer sequence ( $5^{\prime}$ to $3^{\prime}$ ) & Annealing temperature \\
\hline \multirow[t]{2}{*}{$\mathrm{HGF}$} & Forward-CCTTCGAGCTATCGCGGTAAAGAC & $56^{\circ} \mathrm{C}$ \\
\hline & Reverse-TCAAGAGTGTAGCACCATGGCCTC & \\
\hline \multirow[t]{2}{*}{ NGF } & Forward-GGACGCAGCTTTCTATCCTG & $56^{\circ} \mathrm{C}$ \\
\hline & Reverse-GTCCGTGGCTGTGGTCTTAT & \\
\hline \multirow[t]{2}{*}{ GDNF } & Forward-GACTCCAATATGCCCGAAGA & $56^{\circ} \mathrm{C}$ \\
\hline & Reverse-ATGGTAAACCAGGCTGTCGT & \\
\hline \multirow[t]{2}{*}{ BDNF } & Forward-TGGCTGACACTTTTGAGCAC & $56^{\circ} \mathrm{C}$ \\
\hline & Reverse-GCAGCCTTCCTTCGTGTAAC & \\
\hline \multirow[t]{2}{*}{ CNTF } & Forward-TGGCTAGCAAGGAAGATTCG & $56^{\circ} \mathrm{C}$ \\
\hline & Reverse-ACCTTCAAGCCCCATAGCTT & \\
\hline \multirow[t]{2}{*}{ VEGFA } & Forward-ACCAAAGAAAGATAGAACAAAG & $50^{\circ} \mathrm{C}$ \\
\hline & Reverse-GGTGAGAGGTCTAGTTCCCGA & \\
\hline \multirow[t]{2}{*}{ FLK-1 } & Forward-GCCAATGAAGGGGAACTGAAGAC & $50^{\circ} \mathrm{C}$ \\
\hline & Reverse-TCTGACTGCTGGTGATGCTGTC & \\
\hline \multirow[t]{2}{*}{ FGF2 } & Forward-ATCACTTCGCTTCCCGCACT & $55^{\circ} \mathrm{C}$ \\
\hline & Reverse-AGTATGGCCTTCTGTCCAGG & \\
\hline \multirow[t]{2}{*}{ TGF- $\beta 1$} & Forward-CTCTGCAGGCGCAGCTCTG & $55^{\circ} \mathrm{C}$ \\
\hline & Reverse-GGACTCTCCACCTGCAAGAC & \\
\hline \multirow[t]{2}{*}{ IL-10 } & Forward-CAATAACTGCACCCACTTCC & $55^{\circ} \mathrm{C}$ \\
\hline & Reverse-ATTCTTCACCTGCTCCACTGC & \\
\hline \multirow[t]{2}{*}{ TNF- $\alpha$} & Forward-CCCAGACCCTCACACTCAGAT & $55^{\circ} \mathrm{C}$ \\
\hline & Reverse-TTGTCCCTTGAAGAGAACCTG & \\
\hline \multirow[t]{2}{*}{ IL-6 } & Forward-CCGGAGAGGAGACTTCACAG & $55^{\circ} \mathrm{C}$ \\
\hline & Reverse-GAGCATTGGAAGTTGGGGTA & \\
\hline \multirow[t]{2}{*}{ IL-1 $\beta$} & Forward-CAGGAAGGCAGTGTCACTCA & $53^{\circ} \mathrm{C}$ \\
\hline & Reverse-GAAGACAAACCGCTTTTCCA & \\
\hline \multirow[t]{2}{*}{ GAPDH } & Forward-ATGGGAAGCTGGTCATCAAC & $58^{\circ} \mathrm{C}$ \\
\hline & Reverse-GGATGCAGGGATGATGTTCT & \\
\hline
\end{tabular}

HGF, hepatocyte growth factor; NGF, nerve growth factor; GDNF, glial cell line-derived neurotrophic factor; BDNF, brain-derived neurotrophic factor; CNTF, ciliary neurotrophic factor; VEGFA, vascular endothelial growth factor A; FLK-1, vascular endothelial growth factor receptor 2; FGF2, fibroblast growth factor 2; TGF, transforming growth factor; IL, interleukin; TNF, tumor necrosis factor.

China Pharmaceutical Co., Ltd., Shijiazhuang, China) was injected intraperitoneally daily for 4 days postoperatively as prophylaxis against urinary tract infection. In addition, animals were immunosuppressed daily by subcutaneous administration of cyclosporine A (1 mg/100 g; Novartis Pharma Stein AG, Stein, Switzerland) beginning 1 day prior to grafting and continuing until the termination of the experiment.

Gel properties. To examine whether the graft mixture may gelatinize effectively and influence the transplantation process, as well as the gel concentration on cell survival, an extra $7 \mu \mathrm{l}$ graft mixture remaining post-transplantation was added to a $35-\mathrm{mm}$ culture dish and incubated for $45 \mathrm{~min}$ in a $37^{\circ} \mathrm{C} / 5 \% \mathrm{CO}_{2}$ incubator. Subsequently, $1 \mathrm{ml} \mathrm{DMEM} / \mathrm{F}-12$ culture medium containing $10 \%$ FBS was added, and the cells were cultured further.

Small animal in vivo-imaging detection. To examine lesion filling and the survival of DPC/BMSC grafts, the rats ( $\mathrm{n}=3$ at each time point per group) were anesthetized by intraperitoneal injection with $10 \%$ chloral hydrate (400 mg/ml; Sigma-Aldrich; Merck KGaA), then perfused with PBS followed by $4 \%$ paraformaldehyde (Beijing Dingguo Changsheng Biotechnology, Co., Ltd, Beijing, China), on days 14 and 21 post-transplantation, respectively. Subsequently, the spinal cord (2 $\mathrm{cm}$ around the epicenter) was surgically dissected, fixed in $4 \%$ paraformaldehyde at $4^{\circ} \mathrm{C}$ for $48 \mathrm{~h}$, and transferred to $30 \%$ sucrose (Sigma-Aldrich; Merck KGaA) at $4^{\circ} \mathrm{C}$ for $72 \mathrm{~h}$. Finally, the segments of the spinal cords were placed into a new culture dish and the fluorescence intensities around the lesion/graft sites were detected with a Small Animal Imaging system (Xenogen Corporation, Alameda, CA, USA) at $488 \mathrm{~nm}$ excitation.

Assessment of lesion areas. Following fixation and dehydration, the spinal cord segments were embedded with Optimal Cutting Temperature compound (Leica Microsystems $\mathrm{GmbH}$, Wetzlar, Germany), and serial sections of $20-\mu \mathrm{m}$ 
Table II. Primers used for quantitative polymerase chain reaction.

\begin{tabular}{|c|c|}
\hline Gene & Primer sequence (5' to $3^{\prime}$ ) \\
\hline \multirow[t]{2}{*}{ NGF } & Forward-ACCTCTTCGGACACTCTGGA \\
\hline & Reverse-TCCAACCCACACACTGACAC \\
\hline \multirow[t]{2}{*}{ BDNF } & Forward-TGGGTTACACGAAGGAAGGC \\
\hline & Reverse-ATCCTTATGAACCGCCAGCC \\
\hline \multirow[t]{2}{*}{ CNTF } & Forward-GGCAAGCACTGATCGTTGGA \\
\hline & Reverse-TGGAAGGTACGGTAAGCCTG \\
\hline \multirow[t]{2}{*}{$\mathrm{HGF}$} & Forward-ACAGCTTTTTGCCTTCGAGC \\
\hline & Reverse-GCAAGAATTTGTGCCGGTGT \\
\hline \multirow[t]{2}{*}{ GDNF } & Forward-TGACTTGGGTTTGGGCTACG \\
\hline & Reverse-GGTAAACCAGGCTGTCGTCT \\
\hline \multirow[t]{2}{*}{ IL-1 $\beta$} & Forward-TCCATGGTGGATTATGCTCA \\
\hline & Reverse-TTCTGTTCCTGCTCGAGGTT \\
\hline \multirow[t]{2}{*}{ IL-10 } & Forward-ACTGCTAGTTTGCCTGCTCTT \\
\hline & Reverse-ATGTGGGTCTGGCTGACTGG \\
\hline \multirow[t]{2}{*}{ IL-6 } & Forward-CTGCCCTTCAGGAACAGCTATG \\
\hline & Reverse-GGCAGTGGCTGTCAACAACAT \\
\hline \multirow[t]{2}{*}{ TNF- $\alpha$} & Forward-GAGATGTGGAACTGGCAGAGGA \\
\hline & Reverse-TCAGTAGACAGAAGAGCGTGGTG \\
\hline \multirow[t]{2}{*}{ TGF- $\beta 1$} & Forward-CTCCCGTGGCTTCTAGTGC \\
\hline & Reverse-GCCTTAGTTTGGACAGGATCTG \\
\hline \multirow[t]{2}{*}{ VEGFA } & Forward-CCTGGCTTTACTGCTGTACCT \\
\hline & Reverse-GCTGGTAGACGTCCATGAACT \\
\hline \multirow[t]{2}{*}{ GAPDH-1 } & Forward-GAAGGTCGGTGTGAACGGAT \\
\hline & Reverse-ACCAGCTTCCCATTCTCAGC \\
\hline \multirow[t]{2}{*}{ GAPDH-2 } & Forward-ATGGGAAGCTGGTCATCAAC \\
\hline & Reverse-GGATGCAGGGATGATGTTCT \\
\hline
\end{tabular}

HGF, hepatocyte growth factor; NGF, nerve growth factor; GDNF, glial cell line-derived neurotrophic factor; BDNF, brain-derived neurotrophic factor; CNTF, ciliary neurotrophic factor; VEGFA, vascular endothelial growth factor A; FLK-1, vascular endothelial growth factor receptor 2; FGF2, fibroblast growth factor 2; TGF, transforming growth factor; IL, interleukin; TNF, tumor necrosis factor.

thickness were prepared. Every sixth section per rat sample was hydrated in PBS and hematoxylin and eosin staining of the sections was performed. Briefly, the sections were stained with the hematoxylin solution for $5 \mathrm{~min}$, rinsed in running tap water, then differentiated with $1 \%$ hydrochloric acid ethanol for several seconds, followed by rinsing in running tap water. The sections were stained with weak ammonia for 1-2 sec, rinsed in tap water and stained with eosin for $15 \mathrm{~min}$. The sections were dehydrated with ascending concentration gradient of ethyl alcohol (in $80 \%$ ethyl alcohol for 1-2 sec, 95\% ethyl alcohol for $1-2 \mathrm{sec}, 100 \%$ ethyl alcohol I for $5 \mathrm{~min}$ and $100 \%$ ethyl alcohol II for $10 \mathrm{~min})$. The sections were cleared with xylol I for $10 \mathrm{~min}$ and xylol II for $10 \mathrm{~min}$ (Beijing Dingguo Changsheng Biotechnology, Co., Ltd., Beijing, China). All protocols were performed at room temperature. Finally, the sections were mounted with neutral resin and observed under a light microscope at x40 magnification. Finally, the images were captured and merged with Adobe Photoshop CS5 software (version 12.0; Adobe Systems, Inc., San Jose, CA, USA) and cellSens Dimension software (version 1.0; Olympus Corporation, Tokyo, Japan) was used to assess the lesion areas.

Immunocytofluorescence histochemical staining. To assess axonal outgrowth, angiogenesis and inflammation, immunocytofluorescence histochemical staining was conducted in the present study. Briefly, every sixth section per rat sample was hydrated in PBS for 25 min at room temperature; then, samples were permeabilized with $0.1 \%$ Triton X-100 (Sigma-Aldrich; Merck KGaA) for $45 \mathrm{~min}$ at room temperature. Normal goat serum (10\%; Zhongshan Jinqiao Biotechnology Co., Ltd., Beijing, China) was used to block non-specific binding for $1 \mathrm{~h}$ at room temperature. Every sixth section per rat sample was then incubated overnight with primary antibodies at $4^{\circ} \mathrm{C}$ (detailed information regarding the primary antibodies is presented in Table III), followed by incubation with Alexa Fluor ${ }^{\circledR}$ 488-conjugated or 555-conjugated secondary antibodies (1:400; cat. nos. 4412 and 4413; Cell Signaling Technology, Inc., Danvers, MA, USA) for $1 \mathrm{~h}$ at room temperature. Unbound antibody was removed by thoroughly washing with PBS. For the negative control, the primary antibodies were omitted. Finally, the sections were mounted using Anti-Fade mounting medium with DAPI (Vector Laboratories, Inc., Burlingame, CA, USA). All images were obtained using an IX71 fluorescence microscope (Olympus Corporation). The duration of exposure and the parameter settings were identical in $\geq 3$ independent experiments conducted with samples from different groups.

Behavioral testing. Hindlimb motor functions of the DPC-transplant, BMSC-transplant and control group were evaluated weekly by two independent, blinded observers, based on the Basso Beattie Bresnahan (BBB) motor score (14). The number of the rats that received the behavioral test at each time point is presented in Table IV.

Statistical analysis. Statistical analysis was performed using SPSS software, version 17.0 (SPSS, Inc., Chicago, IL, USA). Data are presented as the mean \pm standard error of the mean from $\geq 3$ independent experiments. Multiple-group comparisons were conducted using one-way analysis of variance followed by Least Significant Difference post hoc comparisons (equal variances assumed) or Dunn's post hoc test (unequal variances) for multiple comparisons. Two groups were compared using a Student's t-test. $\mathrm{P}<0.05$ was considered to indicate a statistically significant difference.

\section{Results}

Isolation and cultivation of DPCs and BMSCs from GFP-transgenic rats. After 6 days of cultivation, typical spindle-shaped, fibrocyte-like cells were cultured from the explants, which were all positive for GFP (Fig. 1A and B). The cells demonstrated stable growth following 6 passages; cells revealed fibrocyte-associated morphology and expressed GFP (Fig. 1C and D). As a control, BMSCs were isolated and cultured from the same donor rat. Following culture for 10 days, the clusters consisted of numerous cells that were 
Table III. Primary antibody information.

\begin{tabular}{llll}
\hline Antibody & Dilution & \multicolumn{1}{c}{ Supplier } & Catalogue number \\
\hline GFP & $1: 1,000$ & Abcam (Cambridge, UK) & ab6556 \\
NF-200 & $1: 200$ & EMD Millipore (Billerica, MA, USA) & Mab5262 \\
GFAP & $1: 400$ & Sigma-Aldrich (Merck KGaA, Darmstadt, Germany) & G3893 \\
CD31 & $1: 20$ & Abcam & ab28364 \\
CD11b & $1: 50$ & Bio-Rad Laboratories, Inc., Hercules, CA, USA & MCA275GA \\
\hline
\end{tabular}

GFP, green fluorescent protein; NF, neurofilament; GFAP, glial fibrillary acidic protein; CD, cluster of differentiation.

Table IV. Number of rats that underwent a behavioral test at each time point.

\begin{tabular}{lrrrr}
\hline & \multicolumn{4}{c}{ Day } \\
\cline { 2 - 5 } Group & 0 & 7 & 14 & 21 \\
\hline $\begin{array}{l}\text { Dermal papilla cell-transplant } \\
\text { Bone marrow mesenchymal }\end{array}$ & 10 & 6 & 6 & 3 \\
stem cell-transplant & 9 & 8 & 6 & 3 \\
Control & 8 & 7 & 7 & 4 \\
\hline
\end{tabular}

all positive for GFP (Fig. 1E and F). These controls revealed stable GFP expression after 6 passages (Fig. $1 \mathrm{G}$ and $\mathrm{H}$ ).

Expression of NTFs, angiogenic factors and inflammation-associated cytokines in DPCs. Semi-quantitative RT-PCR analysis revealed that the NTFs, including hepatocyte growth factor (HGF), NGF, GDNF, BDNF and ciliary neurotrophic factor (CNTF) (Fig. 1I); the angiogenic factors, VEGFA (all 3 transcriptional variants) and FGF2 (Fig. 1I); and the inflammation-associated cytokines TGF- $\beta 1$, IL-10, IL-6 and IL-1 $\beta$ (Fig. 1I) were expressed in DPCs at passage 4 (DPC-P4) and passage 7 (DPC-P7), as well as in BMSCs at passage 3 (BMSC-P3) and passage 7 (BMSC-P7). The expression level of the angiogenic factors vascular endothelial growth factor receptor 2 (FLK1) was low in DPCs at passage 4 (DPC-P4) and BMSCs at passage 7 (BMSC-P7). In addition, the expression level of the inflammation-associated cytokine TNF- $\alpha$ was low in DPCs at passage 4.

Additionally, RT-qPCR analysis revealed that, compared with BMSCs, DPCs exhibited significantly higher expression levels of NTFs, BDNF and CNTF, angiogenic factor VEGFA and anti-inflammatory cytokine IL-10. Similar expression levels of NTFs, GDNF and HGF, and anti-inflammatory TGF- $\beta 1$ were observed in DPCs and BMSCs. Significantly lower expression levels of $\mathrm{NGF}$ and pro-inflammatory cytokines TNF- $\alpha$, IL- 6 and IL- $1 \beta$ were observed in DPCs compared with BMSCs (Fig. 1J).

Spinal cord transection and acute injection of cells. T8 vertebra was completely transected and a $\sim 1.5 \mathrm{~mm}$ lesion cavity was created using a combination of iridectomy scissors and microaspiration (Fig. 2A), after which the gap was filled with $7 \mu \mathrm{l}$ DPCs or BMSCs in rat-tail collagen I. Following exposure at room temperature for $45 \mathrm{~min}$, the appearance of the graft mixture became white, translucent and gel-like (Fig. 2B).

Gel-associated properties. Following suspension of the GFP-positive cells in collagen I and culturing in an incubator at $37^{\circ} \mathrm{C}$ for $45 \mathrm{~min}$, the graft mixture gelatinized (data not shown). Subsequently, DMEM/F-12 culture medium supplemented with $10 \%$ FBS was added, and the cells were cultured further. Following overnight growth, most cells in the graft mixture survived and numerous fibrocyte-like cells had begun to migrate from the gel (Fig. 3).

Survival and filling with DPCs/BMSCs grafts. On day 14 (Fig.4A) and 21 (Fig.4B) post-transplantation, the immunofluorescence intensity surrounding the lesion/graft site transplanted with DPCs was stronger compared with the BMSC-transplant group and control group. There was no control group on day 21 as no cell transplanted in the control group on 14 day post transplantation. Additionally, a low-magnification overview of GFP (graft) and glial fibrillary acidic protein (host) immunolabeling on day 21 post-transplantation further revealed that DPCs (Fig. 4C) exhibited greater survival compared with BMSCs (Fig. 4D).

Assessment of lesion areas. On day 21 post-transplantation, the lesion areas of spinal cords ( $n=3$ in each group) transplanted with DPCs (Fig. 5A), BMSCs (Fig. 5B), or collagen alone (Fig. 5C) were $3.79 \pm 0.47,4.90 \pm 1.47$ and $4.52 \pm 0.40 \mathrm{~mm}^{2}$, respectively; thus, no significant difference was observed ( $P>0.05$; Fig. 5D).

Axonal outgrowth. Immunofluorescence staining revealed that axonal growth (arrow) into the lesion sites of the spinal cord transplanted with DPCs (Fig. 6A and B) was greater than that of the BMSC-transplant group (Fig. 6C and D) and control group (Fig. 6E and F) on day 21 post-transplantation. These results indicated that axonal growth in the host may be more notably promoted by transplanted DPCs, and it was associated with positive NF-200 expression and a lack of GFP expression.

Vascular regeneration. Immunofluorescence staining also revealed that vascular regeneration around the lesion sites of spinal cords transplanted with DPCs (Fig. 7A) was greater than that observed in the BMSC-transplant group (Fig. 7B) and the control group (Fig. 7C) on day 21 post-transplantation. 

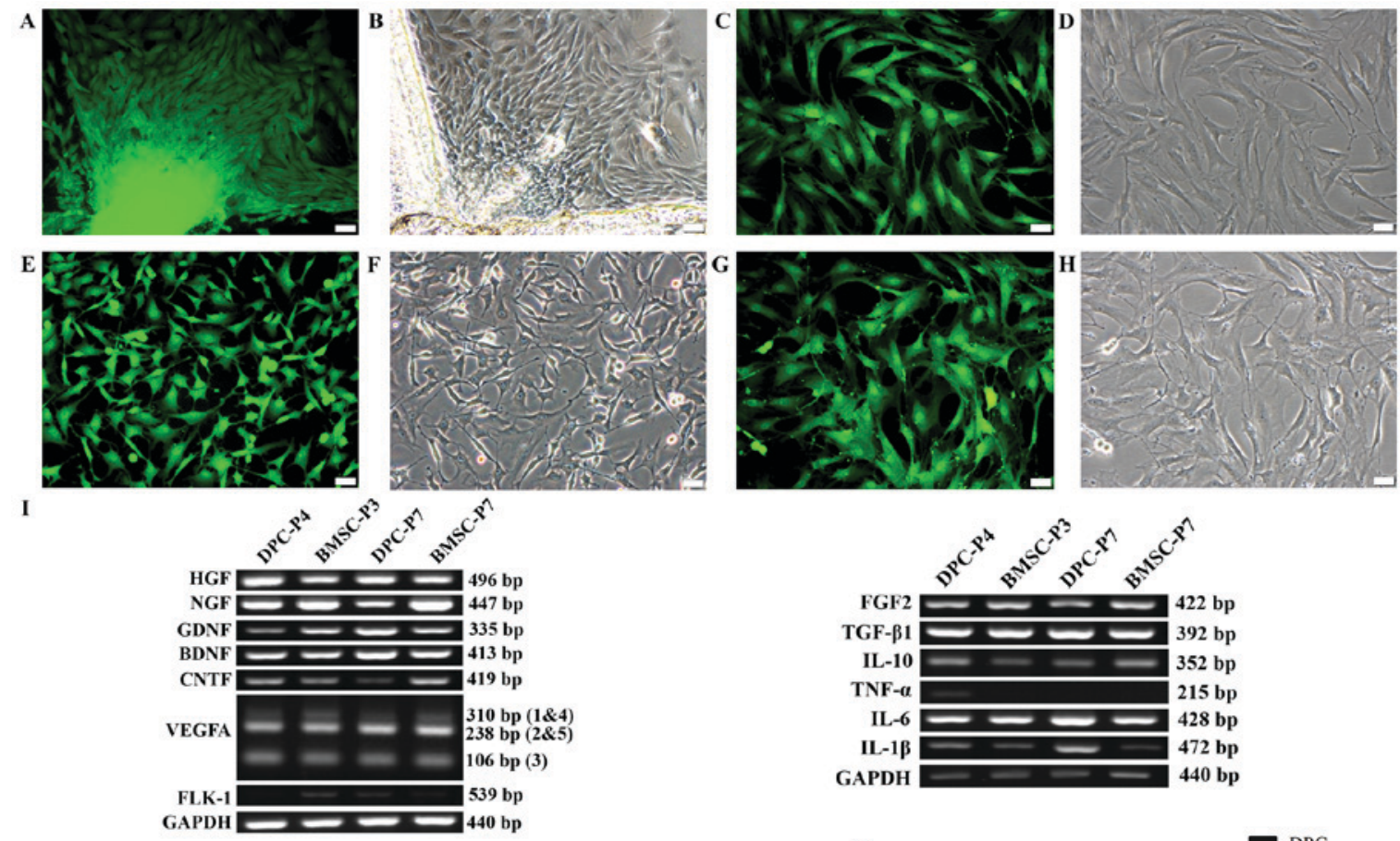

$\mathbf{J}$
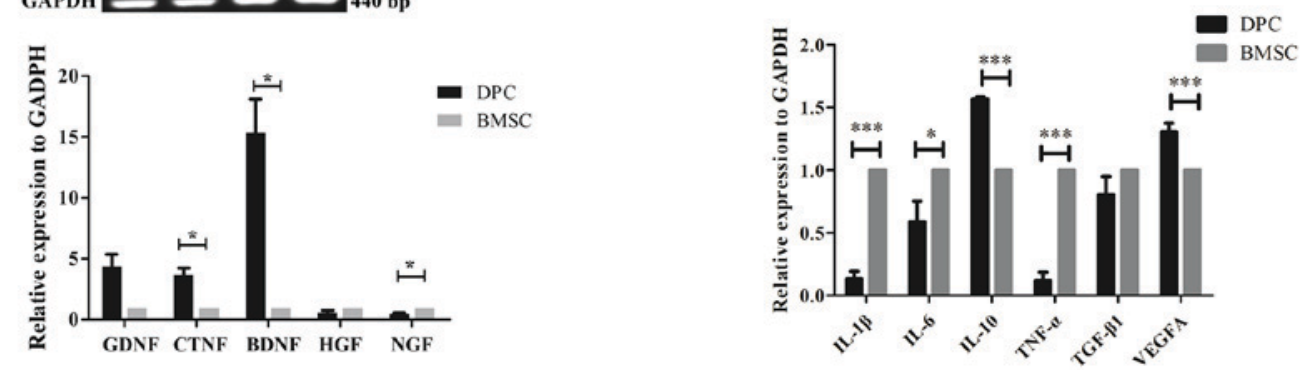

Figure 1. Isolation and cultivation of DPCs and BMSCs from GFP-transgenic rats, and expression of spinal cord injury repair-associated cytokines. (A-G) Isolation and cultivation of DPCs and BMSCs from GFP-transgenic rats. (A) Direct GFP-fluorescence and (B) phase-contrast images demonstrated the morphological characteristics of primary DPCs. (C and D) DPCs at passage 6. (E) Direct GFP-fluorescence and (F) phase-contrast images revealed the morphological characteristics of BMSCs. (G and H) BMSCs at passage 6. (I) Semi-quantitative RT-PCR analysis was performed to confirm the expression of NTFs, angiogenic factors and inflammation-associated cytokines in DPCs. (J) RT-qPCR analysis further revealed relative expression levels of NTFs, angiogenic factors and inflammation-associated cytokines in DPCs. ${ }^{*} \mathrm{P}<0.05,{ }^{* * *} \mathrm{P}<0.001, \mathrm{n}=3$. Scale bars $=20 \mu \mathrm{m}$. BDNF, brain-derived neurotrophic factor; BMSCs, bone marrow mesenchymal stem cells; CNTF, ciliary neurotrophic factor; DPCs, dermal papilla cells; FGF2, fibroblast growth factor 2; FLK1, vascular endothelial growth factor receptor 2; GDNF, glial cell line-derived neurotrophic factor; GFP, green fluorescent protein; HGF, hepatocyte growth factor; IL-1 $\beta$, interleukin-1 $\beta$; IL-6, interleukin-6; IL-10, interleukin-10; NGF, nerve growth factor; NTFs, neurotrophic factors; P, passage number; RT-qPCR, reverse transcription-quantitative polymerase chain reaction; TGF- $\beta 1$, transforming growth factor- $\beta 1$; TNF- $\alpha$, tumor necrosis factor- $\alpha$; VEGFA, vascular endothelial growth factor A.
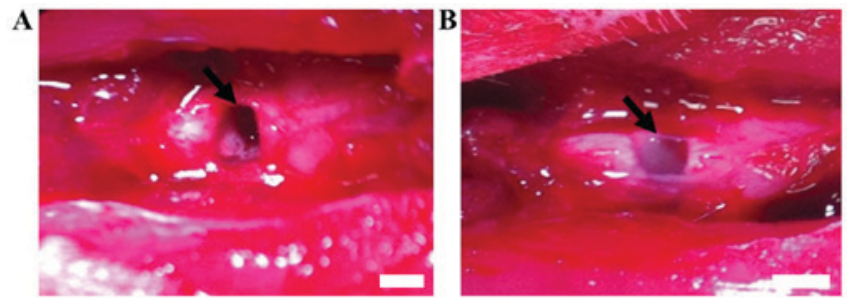

Figure 2. Spinal cord transection and acute injection of dermal papilla cells (A) Pre-transplantation. (B) Post-transplantation. Scale bar=1.5 mm.

These results indicated that DPC transplantation notably promoted revascularization in the host, which was positive for the vascular endothelial cell specific marker CD31 and negative for GFP.

Macrophage infiltration. Immunofluorescence staining revealed that the number of macrophages infiltrating around
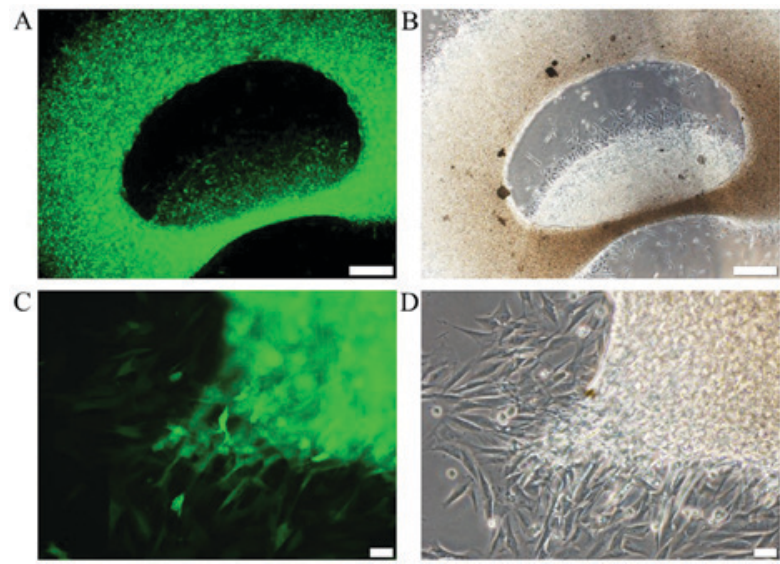

Figure 3. Cultivation of GFP-positive cells suspended in collagen I in vitro. (A) GFP-fluorescence and (B) phase-contrast images demonstrated the survival and migration of GFP-positive cells in the remaining graft mixture following culture in vitro. (C) GFP-fluorescence and (D) phase-contrast images at high magnification. Scale bars: (A and B) $200 \mu \mathrm{m}$, (C and D) $20 \mu \mathrm{m}$. GFP, green fluorescent protein. 

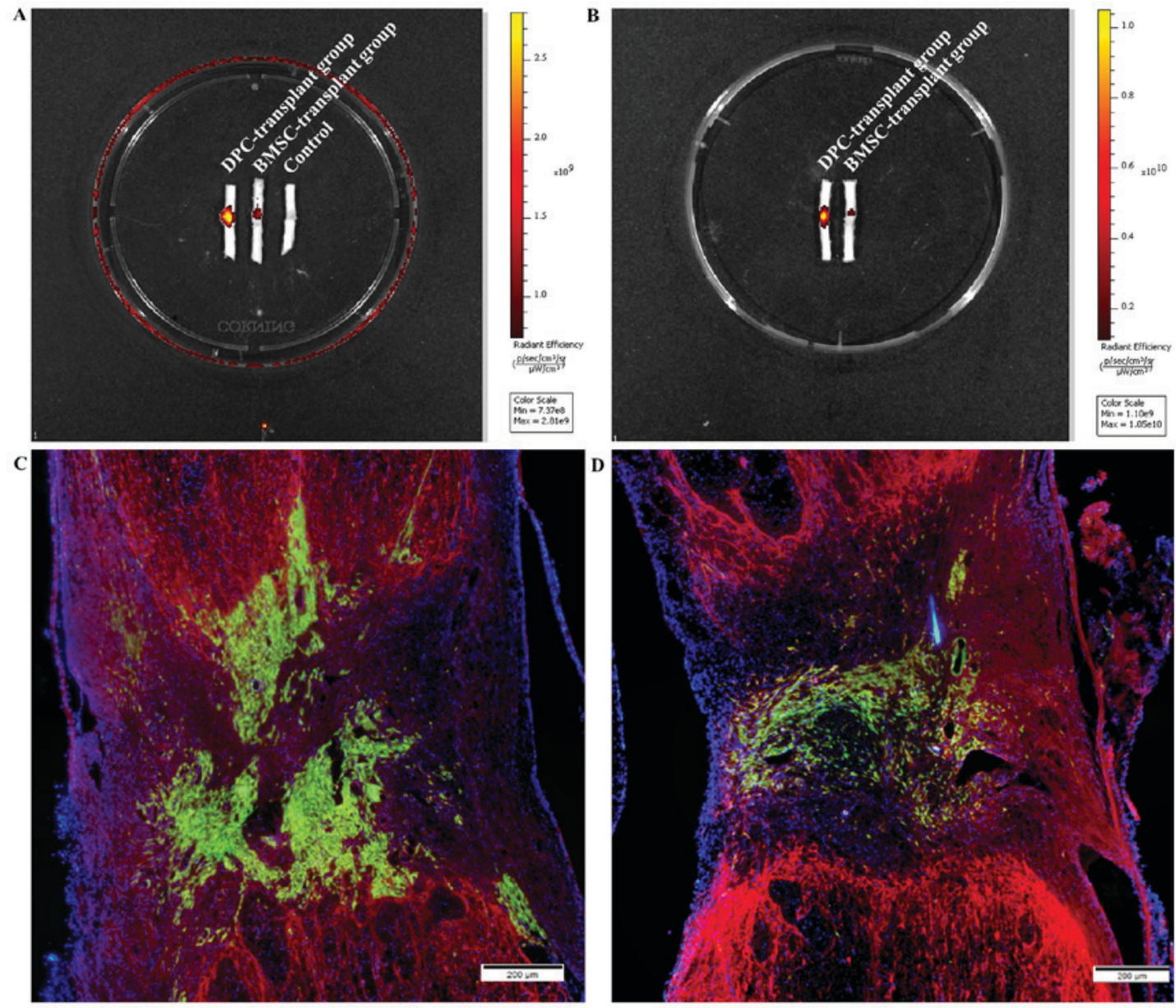

GFP/GFAP/DAPI

GFP/GFAP/DAPI

Figure 4. Survival and filling with DPC and BMSC grafts. Detection of the fluorescence intensity surrounding the lesion/graft site with a small animal-imaging system on (A) day 14 and (B) day 21 post-transplantation. (C and D) Low magnification overview of GFP (graft) and GFAP (host) immunolabeling on day 21 post-transplantation further revealed that (C) DPCs exhibited greater survival compared with (D) BMSCs. Scale bar=200 $\mu \mathrm{m}$. BMSCs, bone marrow mesenchymal stem cells; DPCs, dermal papilla cells; GFAP, glial fibrillary acidic protein; GFP, green fluorescent protein.
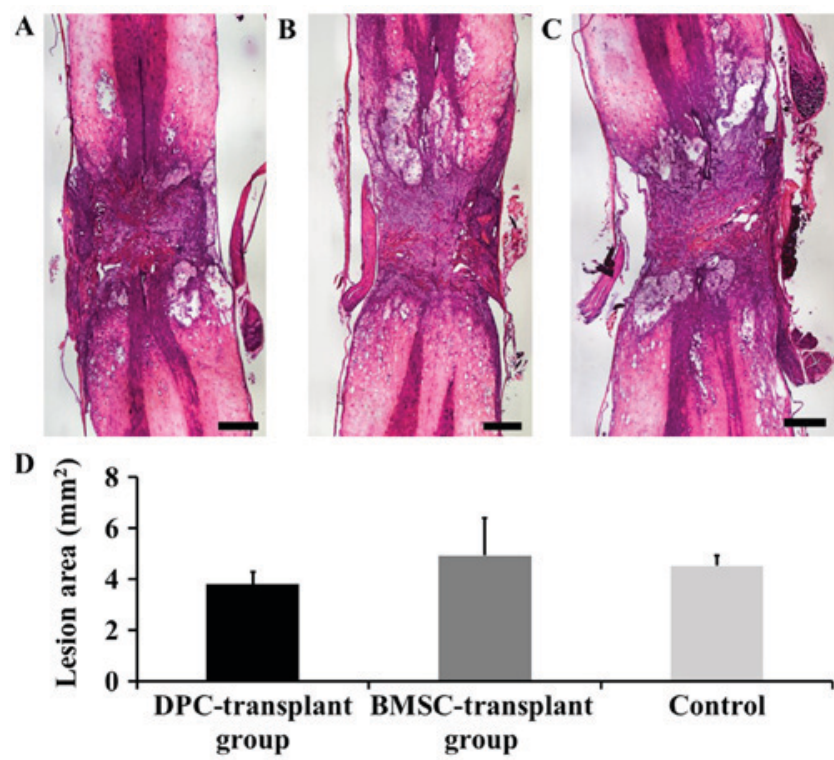

Figure 5. Assessment of lesion areas. Hematoxylin and eosin staining was used to visualize the morphology of the lesion/transplant area in the (A) DPC-transplant group, the (B) BMSC-transplant group and the (C) control group. (D) Quantitative comparative analysis of lesion areas. $\mathrm{P}>0.05$. Scale bar $=500 \mu \mathrm{m}$. BMSCs, bone marrow mesenchymal stem cells; DPCs, dermal papilla cells. the lesion sites of spinal cords transplanted with DPCs was greater than that observed in the BMSC-transplant group (Fig. 8), as determined by CD11b staining.

Behavioral testing. On days 0, 7, 14 and 21 post-transplantation, the BBB scores of the DPC-transplant, BMSC-transplant and control groups were all 0 , indicating that no functional improvement occurred (data not shown).

\section{Discussion}

The combined data from the present and a previous study reveal that, similar to BMSCs, DPCs express and secrete various cytokines and growth factors, which promote the reconstruction of a supportive microenvironment for neural regeneration in SCI lesion sites following transplantation (11). However, the therapeutic functions of DPCs were more favorable than BMSCs, which further indicated that DPCs may be a valuable alternative source of stem cells for autologous cell therapy to treat SCI.

Stem cells, which are self-renewing and highly multipotent, are potentially useful for repairing neuronal functions following SCI. According to previous reports, BMSC transplantation is an effective method for treating SCI $(15,16)$, but BMSCs must be isolated by aspiration, which is traumatic 


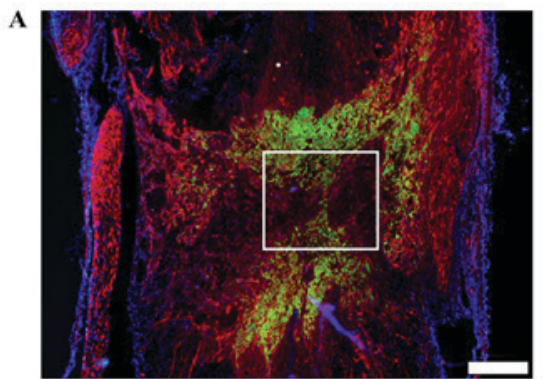

GFP/NF-200/DAPI

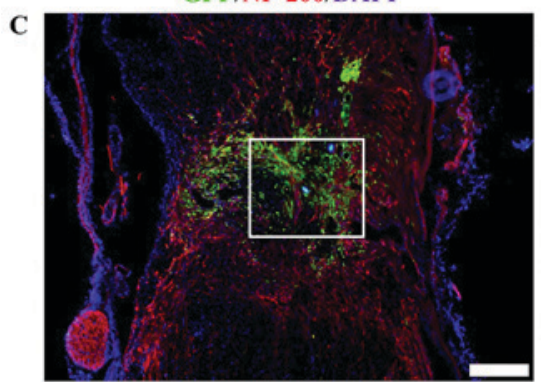

GFP/NF-200/DAPI

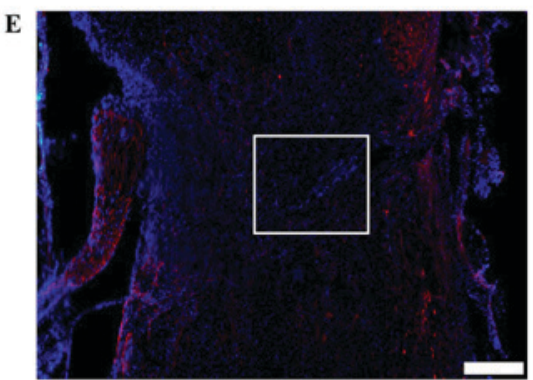

GFP/NF-200/DAPI

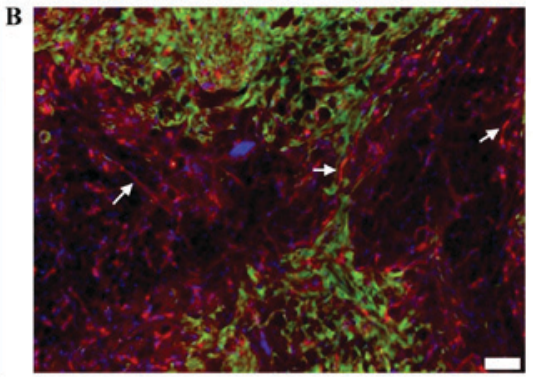

GFP/NF-200/DAPI

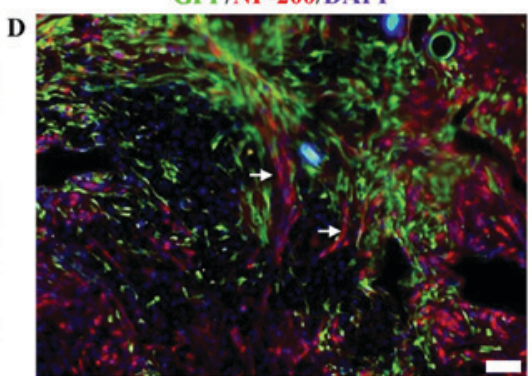

GFP/NF-200/DAPI

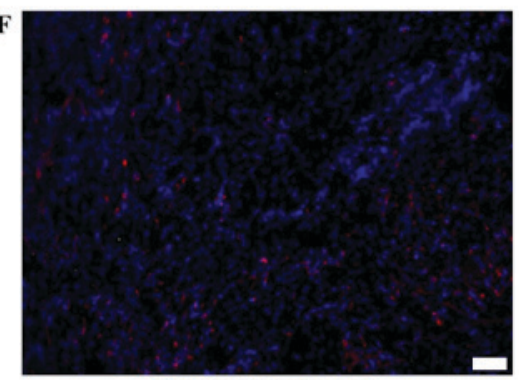

GFP/NF-200/DAPI

Figure 6. DPCs promote axonal outgrowth into the T8 vertebra complete transection site. Immunofluorescence staining revealed that axonal outgrowth into lesion sites of the spinal cord in the (A and B) DPC-transplant group was greater than that of the (C and D) BMSC-transplant group and the (E and F) control group, as determined by NF-200 staining and analysis of GFP fluorescence. The right hand panel indicates higher magnification images of the left hand panel. White arrow, axonal outgrowth positive for NF-200. Scale bars: $200 \mu \mathrm{m}$ (A, C and E) and $27 \mu \mathrm{m}$ (B, D and F). BMSCs, bone marrow mesenchymal stem cells; DPCs, dermal papilla cells; GFP, green fluorescent protein; NF, neurofilament.

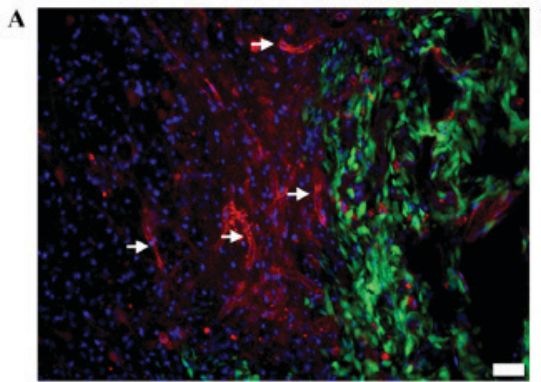

CD31/GFP/DAPI

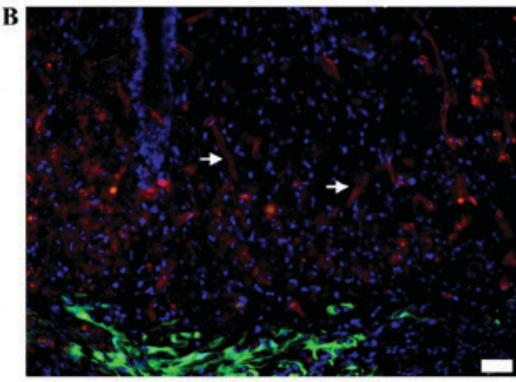

CD31/GFP/DAPI

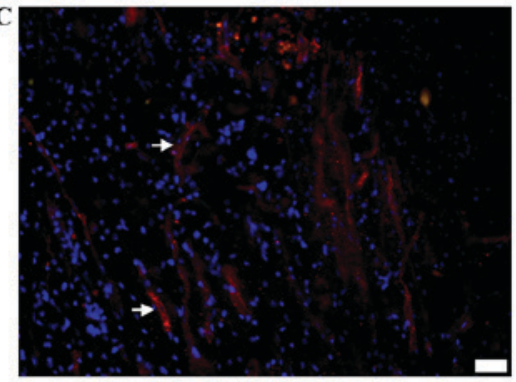

CD31/GFP/DAPI

Figure 7. DPCs promote vascular regeneration around lesion sites. Immunofluorescence staining revealed that vascular regeneration (white arrows) around lesion sites of spinal cords transplanted with (A) dermal papilla cells was greater than that observed with the (B) bone marrow mesenchymal stem cell-transplant group and the (C) control group, as determined by CD31 staining. Scale bars $=20 \mu \mathrm{m}$. CD31, cluster of differentiation 31; GFP, green fluorescent protein.

and painful (17). In addition, the percentage of BMSCs in the bone marrow decreases with age, as do their differentiation abilities (18). Conversely, DPCs can be isolated by directly removing hairs, which is a non-traumatic procedure, and hair follicles are renewable organs. Furthermore, hair undergoes repeated cycles of growth (anagen phase), regression (catagen phase) and rest (telogen phase) throughout the lives of mammals $(19,20)$. In addition, we previously demonstrated that DPCs, with partial properties of neural crest stem cells, secrete greater amounts of BDNF and GDNF compared with BMSCs (11). Thus, the abilities of DPCs to promote SCI repair in vivo were compared in the present study.

At present, numerous types of SCI models have been established, including the impact-injury model, the crush-injury model, and the complete transection and aspiration-injury model $(13,21,22)$. Among these, the complete transection and 

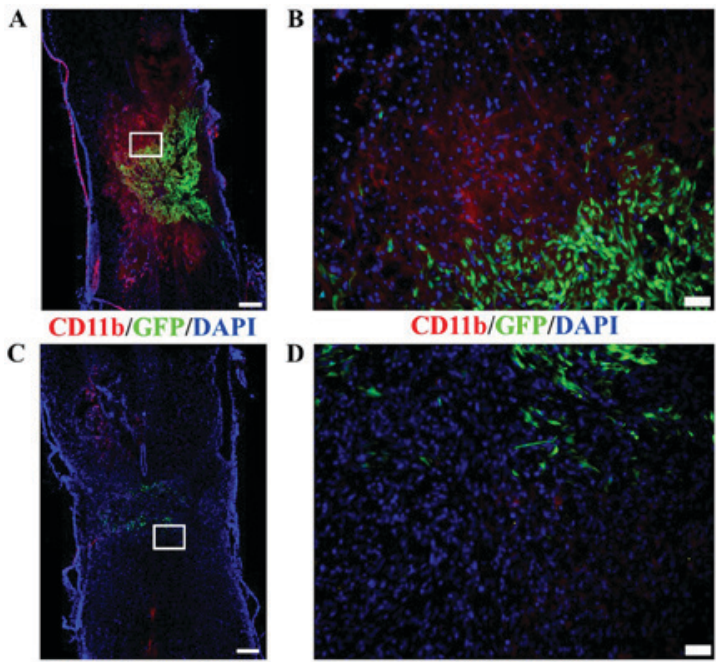

CD11b/GFP/DAPI

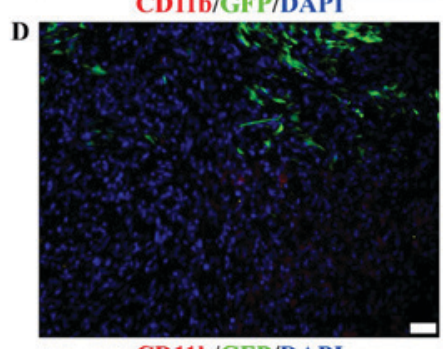

CD11b/GFP/DAPI

Figure 8. Macrophage infiltration. Immunofluorescence staining revealed that macrophages infiltration surrounding lesion sites of spinal cords transplanted with (A and B) dermal papilla cells was greater than that observed in the (C and $\mathrm{D})$ bone marrow mesenchymal stem cell-transplant group, as determined by CD11b staining. The right hand panel indicates higher magnification images of the left hand panel. Scale bars: (A and C) $200 \mu \mathrm{m}$, (B and D) $20 \mu \mathrm{m}$. CD11b, cluster of differentiation molecule 11b; GFP, green fluorescent protein.

aspiration-injury model does not require expensive and complicated equipment, and may be easily generated via relatively simple surgical process. Therefore, it has been widely used to study axonal regeneration and outgrowth at injury sites. For these reasons, this SCI model was selected for analysis in the present study.

Biomaterial scaffolds provide a bridge to connect lost tissues, an adhesion site for implanted or host cells, thus are widely used in the research of SCI repair via stem cell transplantation $(23,24)$. Fibronectin, as a carrier of NTFs, has been reported to provide a suitable axon-growth environment within rat SCI sites (25); however, fibronectin is expensive and the solidity of the gel formed by mixing fibrinogen with thrombin is hard to control. Furthermore, the distribution of cells mixed with fibronectin is nonuniform. By contrast, collagen I possesses the properties of good biocompatibility, biodegradability and low immunogenicity, and has been widely used in tissue-regeneration research (26). In addition, particular concentrations of collagen I remain at a liquid state at $4^{\circ} \mathrm{C}$ and form a solid scaffold of particular physical strength at $37^{\circ} \mathrm{C}$, which integrates well with peripheral tissue, exhibits good plasticity post-transplantation (27). This results in uniform distribution of the cells suspended with collagen for transplantation, provides mechanical support for transplanted cells and provides a highly porous network structure that allows for cell expansion, neurite extension, and transport of nutrients, all of which are essential for cell survival (27). Thus, collagen I served as a scaffold in the present study. Additionally, collagen I, as a promising three-dimensional cell culture scaffold, maintains the survival and migration ability of NPSCs under optimal conditions $(0.5-0.75 \mathrm{mg} / \mathrm{ml})(27)$. In the present study, it was also observed that the dispersion and nonuniform distribution of the cells transplanted was effectively avoided by using $2 \mathrm{mg} / \mathrm{ml}$ collagen I as scaffold.
Immediately following a traumatic event, the microenvironment of the spinal cord undergoes acute inflammation, which is unfavorable for the survival of transplants. This factor is almost always a barrier for cell-based therapy in the acute phase, but in the present study, the DPCs survived well in the lesion site of spinal cord, and the amount was greater than that of BMSCs. In addition, the number of macrophages infiltrated in the DPC-transplanted group was greater compared with the BMSC-transplanted group. Based on the expression of inflammation-associated cytokines (IL-10 and TGF- $\beta 1$ ), which have important roles in regulating the polarization of pro-inflammatory M1-type macrophages into anti-inflammatory M2-type macrophages $(28,29)$, DPCs may have modified the inflammatory environment by regulating the conversion of M1 to M2 macrophages. The regulatory effects of DPCs on polarization of macrophages from M1 to M2 phenotype may be investigated in the future.

Liu et al (30) reported that nestin-positive dermal and bulge area cells may differentiate into neuronal and glial cells after transplantation to injured spinal cords. Conversely, DPC differentiation into neurons or astrocytes was not observed in the present study; the cell cultivation conditions used may have influenced the cellular differentiation ability. Generally, BMSCs are expanded via culturing in conventional monolayers. To confirm the therapeutic effect of DPCs on promoting SCI repair under the same culture condition used with BMSCs, DPCs were also expanded in monolayers, instead of in serum-free medium supplemented with epidermal growth factor and bFGF. Culturing DPCs in monolayers may have altered the differentiation abilities of nestin-positive NCSCs to differentiate into DPCs, thereby inhibiting their capacity for differentiation into neuronal and glial cells in SCI lesion sites. Furthermore, the results of the present study revealed that, similar to BMSCs, DPCs may also stimulate neural tissue regeneration, primarily via the secretion of numerous cytokines and growth factors. Although DPCs differentiated into neurons, the rate of differentiation may not have been high enough to serve a crucial function during the repair of SCI.

The SCI environment tends to promote the astrocytic differentiation of neural stem cells (NSCs). Lu et al (25) reported that NSCs embedded into fibrin matrices containing BNDF and GDNF, which differentiated into numerous cellular phenotypes following transplantation into severe SCI sites and subsequently stimulated axonal outgrowth effectively. In a previous study by our group, DPCs actively secrete BDNF and GDNF compared with BMSCs, and notably induce the neuronal differentiation of $\mathrm{PC} 12$ cells and promote the outgrowth of neuritis (11). Thus, following transplantation, DPCs may secrete BDNF and GDNF to form a more beneficial microenvironment for the repair of SCI lesions, which eventually promote axon sprouting.

In the present study, it was also observed that angiogenesis in the DPC-transplanted group was markedly pronounced. Based on the higher expression levels of angiogenic factor VEGFA in DPCs, the promotion of angiogenesis in the DPC-transplanted group may be due to the secretion of the VEGFA.

Additionally, histological repair was also observed, including enhanced lesion filling; more axons formed within 
the lesion sites with DPC grafts; however, the axons did not completely develop into the lesion site so as to form axonal connections with the caudal end during the short observation period. Thus, the recovery of hindlimb motor function with BBB testing was not observed in either the DPC-transplant group or the BMSC-transplant group. In the future, the in vivo experimental period may be extended to 3-6 months to further examine cell fates, the formation of neural networks across sites of complete spinal transection and the recovery of motor functions.

In summary, the data of the present study demonstrated that DPCs promote greater tissue repair following complete SCI compared with BMSCs, including the promotion of neurite outgrowth and greater revascularization following the secretion of neurotrophic factors, angiogenic factors and inflammation-associated cytokines. The findings of the present study suggest that these cells may be a promising resource for autologous cell therapy in the treatment of SCIs.

\section{Acknowledgements}

Not applicable.

\section{Funding}

The present study was supported by the National Natural Science Foundation of China (grant no. 81572139), the National Natural Science Foundation of China (grant no. 81571199), the Young Teachers' Innovation Projects from Jilin University (grant no. 450060521193), the Bethune Project from Jilin University (grant no. 2015430) and the Outstanding Young Teacher's Training Program from Jilin University (grant no. 419080500576).

\section{Availability of data and materials}

The datasets used and/or analyzed during the current study are available from the corresponding author on reasonable request.

\section{Authors' contributions}

ML and XM isolated and cultured dermal papilla cells and bone marrow mesenchymal stem cells, performed reverse transcription-polymerase chain reaction, assayed the gel properties, assessed the lesion areas, performed immunocytofluorescence histochemical staining, analyzed data and wrote the manuscript. SL and JinX performed acute injections of the cells. ZZ and DS performed behavioral testing. JiaX and $\mathrm{XH}$ performed reverse transcription-quantitative polymerase chain reaction. GC designed the study, established the rat models of spinal injury and performed small animal in vivo imaging. YL designed the study and provided research funding.

\section{Ethics approval and consent to participate}

The present study was approved by the Ethics Committee of Jilin University (Changchun, China) and conformed to their regulatory standards.

\section{Consent for publication}

Not applicable.

\section{Competing interests}

The authors declare that they have no competing interests.

\section{References}

1. Nakamura $\mathrm{M}$ and Okano $\mathrm{H}$ : Cell transplantation therapies for spinal cord injury focusing on induced pluripotent stem cells. Cell Res 23: 70-80, 2013.

2. Tang L, Lu X, Zhu R, Qian T, Tao Y, Li K, Zheng J, Zhao P, Li S, Wang X and Li L: Adipose-derived stem cells expressing the neurogenin-2 promote functional recovery after spinal cord injury in rat. Cell Mol Neurobiol 36: 657-667, 2016.

3. Mothe AJ and Tator $\mathrm{CH}$ : Advances in stem cell therapy for spinal cord injury. J Clin Invest 122: 3824-3834, 2012.

4. Yokota K, Kobayakawa K, Kubota K, Miyawaki A, Okano H, Ohkawa Y, Iwamoto Y and Okada S: Engrafted neural stem/progenitor cells promote functional recovery through synapse reorganization with spared host neurons after spinal cord injury. Stem Cell Reports 5: 264-277, 2015.

5. Wang YX, Sun JJ, Zhang M, Hou XH, Hong J, Zhou YJ and Zhang ZY: Propofol injection combined with bone marrow mesenchymal stem cell transplantation better improves electrophysiological function in the hindlimb of rats with spinal cord injury than monotherapy. Neural Regen Res 10: 636-643, 2015.

6. Sabapathy V, Tharion G and Kumar S: Cell therapy augments functional recovery subsequent to spinal cord injury under experimental conditions. Stem Cells Int 2015: 132172, 2015.

7. Neirinckx V, Cantinieaux D, Coste C, Rogister B, Franzen R and Wislet-Gendebien S: Concise review: Spinal cord injuries: How could adult mesenchymal and neural crest stem cells take up the challenge? Stem Cells 32: 829-843, 2014.

8. Ritfeld GJ, Patel A, Chou A, Novosat TL, Castillo DG, Roos RA and Oudega M: The role of brain-derived neurotrophic factor in bone marrow stromal cell-mediated spinal cord repair. Cell Transplant 24: 2209-2220, 2015.

9. Cantinieaux D, Quertainmont R, Blacher S, Rossi L, Wanet T, Noël A, Brook G, Schoenen J and Franzen R: Conditioned medium from bone marrow-derived mesenchymal stem cells improves recovery after spinal cord injury in rats: An original strategy to avoid cell transplantation. PLoS One 8: e69515, 2013

10. Wang X, Zou X, Zhao J, Wu X, E L, Feng L, Wang D, Zhang G, Xing $\mathrm{H}$ and Liu H: Site-specific characteristics of bone marrow mesenchymal stromal cells modify the effect of aging on the skeleton. Rejuvenation Res 2016 Mar 15 (Epub ahead of print).

11. Li M, Liu JY, Wang S, Xu H, Cui L, Lv S, Xu J, Liu S, Chi G and Li Y: Multipotent neural crest stem cell-like cells from rat vibrissa dermal papilla induce neuronal differentiation of PC12 cells. Biomed Res Int 2014: 186239, 2014.

12. Livak KJ and Schmittgen TD: Analysis of relative gene expression data using real-time quantitative PCR and the 2(-Delta Delta C(T)) method. Methods 25: 402-408, 2001.

13. Medalha CC, Jin Y, Yamagami T, Haas C and Fischer I: Transplanting neural progenitors into a complete transection model of spinal cord injury. J Neurosci Res 92: 607-618, 2014.

14. Molina AE, Cristante AF, de Barros TE Sr, Molina MS and Molina TP: A computerized system for the application of Basso, Beattie and Bresnahan scale in Wistar rats. Acta Ortop Bras 23: 179-183, 2015.

15. Nakano N, Nakai Y, Seo TB, Homma T, Yamada Y, Ohta M, Suzuki Y, Nakatani T, Fukushima M, Hayashibe M and Ide C: Effects of bone marrow stromal cell transplantation through CSF on the subacute and chronic spinal cord injury in rats. PLoS One 8: e73494, 2013.

16. Dasari VR, Veeravalli KK and Dinh DH: Mesenchymal stem cells in the treatment of spinal cord injuries: A review. World J Stem Cells 6: 120-133, 2014.

17. Klar AS, Zimoch J and Biedermann T: Skin tissue engineering: Application of adipose-derived stem cells. Biomed Res Int 2017: 9747010, 2017. 
18. Li C, Wei G, Gu Q, Wen G, Qi B, Xu L and Tao S: Donor age and cell passage affect osteogenic ability of rat bone marrow mesenchymal stem cells. Cell Biochem Biophys 72: 543-549, 2015.

19. Hoffman RM: The hair follicle as a gene therapy target. Nat Biotechnol 18: 20-21, 2000.

20. Driskell RR, Clavel C, Rendl M and Watt FM: Hair follicle dermal papilla cells at a glance. J Cell Sci 124: 1179-1182, 2011.

21. Tyor WR, Avgeropoulos N, Ohlandt G and Hogan EL: Treatment of spinal cord impact injury in the rat with transforming growth factor-beta. J Neurol Sci 200: 33-41, 2002.

22. Walker MJ, Walker CL, Zhang YP, Shields LB, Shields CB and Xu XM: A novel vertebral stabilization method for producing contusive spinal cord injury. J Vis Exp 5: e50149, 2015.

23. Kim M, Park SR and Choi BH: Biomaterial scaffolds used for the regeneration of spinal cord injury (SCI). Histol Histopathol 29: 1395-1408, 2014.

24. Assunção-Silva RC, Gomes ED, Sousa N, Silva NA and Salgado AJ: Hydrogels and cell based therapies in spinal cord injury regeneration. Stem Cells Int 2015: 948040, 2015.

25. Lu P, Wang Y, Graham L, McHale K, Gao M, Wu D, Brock J, Blesch A, Rosenzweig ES, Havton LA, et al: Long-distance growth and connectivity of neural stem cells after severe spinal cord injury. Cell 150: 1264-1273, 2012.

26. Nectow AR, Marra KG and Kaplan DL: Biomaterials for the development of peripheral nerve guidance conduits. Tissue Eng Part B Rev 18: 40-50, 2012.
27. Watanabe $K$, Nakamura $M$, Okano $H$ and Toyama $Y$ : Establishment of three-dimensional culture of neural stem/progenitor cells in collagen Type-1 Gel. Restor Neurol Neurosci 25: 109-117, 2007.

28. Boehler RM, Kuo R, Shin S, Goodman AG, Pilecki MA, Gower RM, Leonard JN and Shea LD: Lentivirus delivery of IL-10 to promote and sustain macrophage polarization towards an anti-inflammatory phenotype. Biotechnol Bioeng 111: 1210-1221, 2014

29. Zhang F, Wang $H$, Wang $X$, Jiang G, Liu H, Zhang G, Wang H, Fang $\mathrm{R}, \mathrm{Bu} \mathrm{X}$, Cai $\mathrm{S}$ and Du J: TGF- $\beta$ induces M2-like macrophage polarization via SNAIL-mediated suppression of a pro-inflammatory phenotype. Oncotarget 7: 52294-52306, 2016.

30. Liu F, Uchugonova A, Kimura H, Zhang C, Zhao M, Zhang L, Koenig K, Duong J, Aki R, Saito N, et al: The bulge area is the major hair follicle source of nestin-expressing pluripotent stem cells which can repair the spinal cord compared to the dermal papilla. Cell Cycle 10: 830-839, 2011.

This work is licensed under a Creative Commons Attribution-NonCommercial-NoDerivatives 4.0 International (CC BY-NC-ND 4.0) License. 